Supporting Information

\title{
Long-Chain Chlorinated Paraffins Have Reached the Arctic
}

Bo Yuan, ${ }^{1, *}$ Michael S. McLachlan, ${ }^{1}$ Anna M. Roos, ${ }^{2,3}$ Malene Simon, ${ }^{2}$ Anna Strid, ${ }^{4}$ and Cynthia A. de Wit ${ }^{1}$

${ }^{1}$ Department of Environmental Science, Stockholm University, 10691 Stockholm, Sweden

${ }^{2}$ Greenland Climate Research Center, Greenland Institute of Natural Resources, 3900 Nuuk, Greenland

${ }^{3}$ Department of Environmental Research and Monitoring, Swedish Museum of Natural History, 10405 Stockholm, Sweden

${ }^{4}$ RISE Research Institutes of Sweden, 11486 Stockholm, Sweden

* Corresponding author address and e-mail:

Svante Arrhenius väg 8, SE-10691 Stockholm, Sweden; bo.yuan@aces.su.se.

Pages: 11; Text: 4; Figure: 1; Tables: 4. 


\section{Ethical permissions}

CITES permissions were obtained to send samples from Greenland to Sweden, certificate numbers: 20GL1717528 (harbor porpoise), 20GL1717530 (killer whale), 20GL1717531 (narwhals), 20GL1717532 (pilot whales), and 17GL1167089 (minke whales). The cetacean samples from Sweden were subsampled from the Environmental Specimen Bank at Swedish Museum of Natural History. They were from individuals that were either stranded or bycaught in fishing gear.

\section{Instrument method}

UPLC-APCI-Orbitrap-HRMS (Q Exactive, Thermo Fisher Scientific, San Jose, USA) was operated in full-scan mode (m/z 250-2000) with a resolution of 120000 FWHM. An ACQUITY UPLC BEH C18 column $(1.7 \mu \mathrm{m}, 2.1 \times 50 \mathrm{~mm}$, Waters, Manchester, U.K.) was maintained at $40{ }^{\circ} \mathrm{C}$, the injection volume was $3 \mu \mathrm{L}$, and the flow rate was $0.4 \mathrm{~mL} / \mathrm{min}$. The mobile phases consisted of water (A) and methanol (B). The gradient elution started from $10 \%$ B for $0.5 \mathrm{~min}$, ramped to $100 \%$ by $2.5 \mathrm{~min}$, held $2.5 \mathrm{~min}$, ramped to $10 \%$ by $5.1 \mathrm{~min}$, and finally held for $1 \mathrm{~min}$. Dichloromethane (DCM) was introduced into the mobile phase at a flow rate of $0.028 \mathrm{~mL} / \mathrm{min}$ between the UPLC column and the ion source using a syringe pump. The instrumental settings were set as follows: DCM flow rate $0.028 \mathrm{~mL} / \mathrm{min}$, capillary temperature $250{ }^{\circ} \mathrm{C}$, Aux (auxiliary) gas heater temperature $250{ }^{\circ} \mathrm{C}$, spray current $5.7 \mu \mathrm{A}$, maximum IT (ion time) $250 \mathrm{~ms}$, AGC (automatic gain control) target 5e6, sheath gas flow rate 17 arbs, and Aux gas flow rate 1 arb.

\section{Quantification of CPs}

Quantification of vSCCPs, SCCPs, MCCPs, and LCCPs was made based on a $\mathrm{C}_{n} \mathrm{Cl}_{m^{-}}$ homologue profile reconstruction method ${ }^{1}$ and has been introduced in detail in Du et al. ${ }^{2}$ The CP profile of each sample was reconstructed by linearly superimposing the profiles of several $\mathrm{CP}$ reference standards. Eighteen CP commercial mixtures were used for quantification (Tables $\mathrm{S} 3$ and S4). The performance of quantification was evaluated with the goodness-of-fit $\mathrm{R}^{2}$ between the measured $\mathrm{C}_{n} \mathrm{Cl}_{m}$ profile and the linearly superimposed one. The single-chainlength standards were used in order to improve the performance of profile reconstruction ${ }^{3,4}$ and the comparability of CP concentrations with different instrumental setup and quantification methods. ${ }^{5}$ Quantification of S/M/LCCPs in most samples fulfilled the criterion of $\mathrm{R}^{2} \geq 0.50$ (Table S3), ${ }^{1}$ which suggested a mean deviation of concentration less than $40 \% .{ }^{6}$ The two results with $\mathrm{R}^{2}<0.5$ were reported as tentative values. ${ }^{7}$ For quantification of vSCCPs, the $\mathrm{R}^{2}$ ranged from 0.34-0.83 (median: 0.53; Table S4).

\section{Migration of the sampled species and their food supply}

Blue mussel and Iceland scallop are not migratory. Greenland sharks are resident Arctic species. Some Greenland sharks have been tagged so it is known that they can swim from west Greenland to Iceland and back. Minke whales feed in the Arctic before they migrate south for the winter and mating. However, some minke whales may stay in the sea ice free Arctic during winter as well. Narwhals stay in the Arctic. They feed in the middle of Baffin Bay during winter. ${ }^{8}$ The migrations of killer whales and pilot whales from Greenland are not clear to date. However, based on knowledge from these species in other areas, the best guess is that they do not migrate much. If they migrate, it can be because they follow a certain prey species, such as 
mackerel. In that case, the mackerel would migrate between Greenland and Icelandic waters. The Swedish porpoise in this study originates from the Belt Sea population, and tagging data on them suggest they stay in the Scandinavian part of Europe between Denmark and Sweden. ${ }^{9}$ The Greenland porpoise may migrate long-distance within the North Atlantic and return to West Greenland to breed. ${ }^{10}$

$\square \mathrm{Cl}_{2} \square \mathrm{Cl}_{3} \square \mathrm{Cl}_{4} \square \mathrm{Cl}_{5} \square \mathrm{Cl}_{6} \quad \square \mathrm{Cl}_{7} \square \mathrm{Cl}_{8} \quad \square \mathrm{Cl}_{9} \square \mathrm{Cl}_{10} \square \mathrm{Cl}_{11} \square \mathrm{Cl}_{12}$

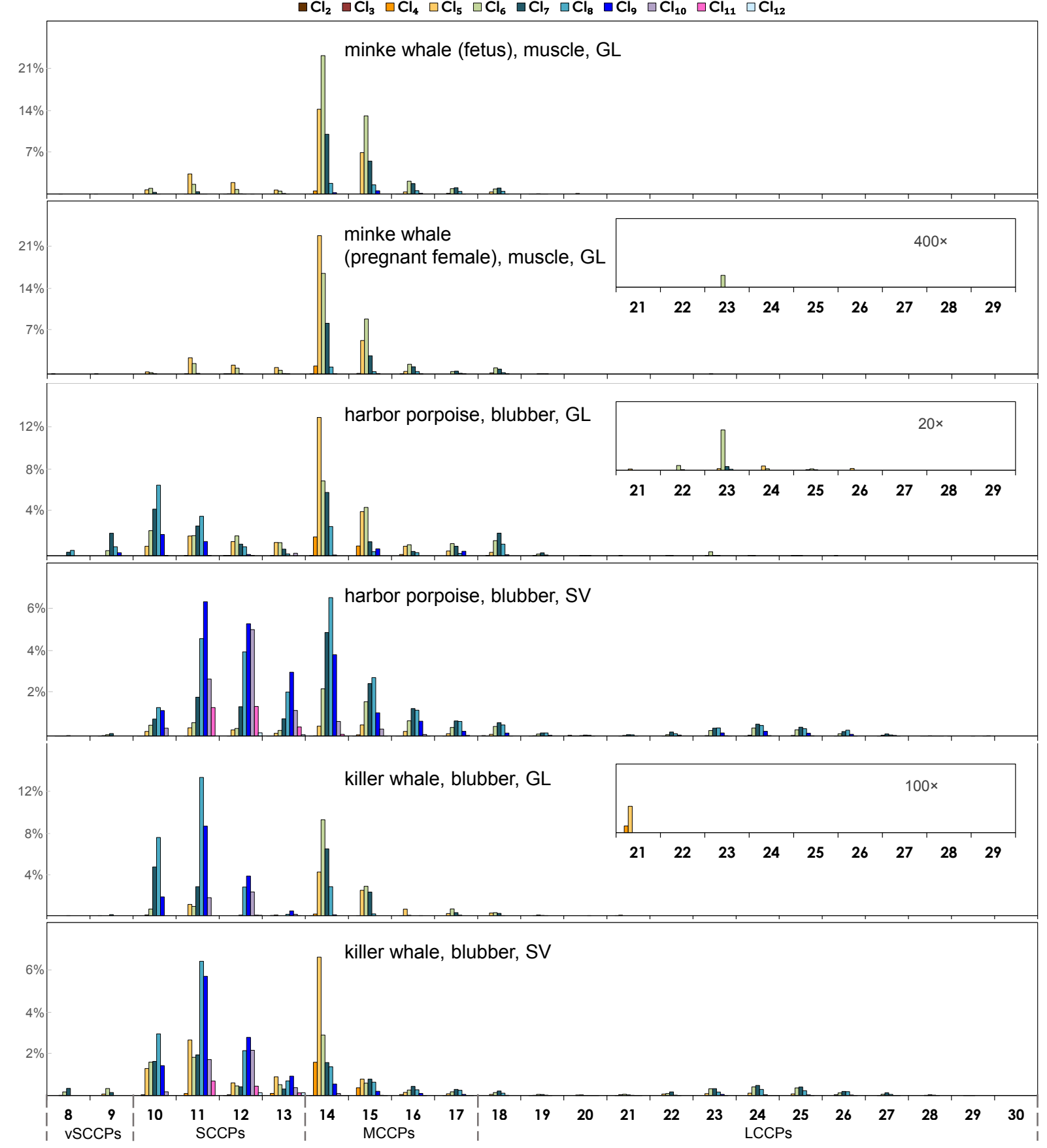

Figure S1. Relative abundance of CP carbon-chlorine homologues in marine samples collected from Greenland (GL) and Sweden (SV), with enlarged display of $\mathrm{C}_{21-29} \mathrm{CPs}$. The horizontal axes represent carbon chain length. 
Table S1. Concentrations and method detection limits (MDLs) of CPs in individual samples.

\begin{tabular}{|c|c|c|c|c|c|c|c|c|c|c|c|c|c|}
\hline \multirow{2}{*}{ species } & \multirow{2}{*}{ tissue } & \multirow{2}{*}{ site } & \multirow{2}{*}{ year } & \multirow{2}{*}{ sample info } & \multicolumn{4}{|c|}{ Concentration (ng/g lipid) } & \multirow{2}{*}{$\begin{array}{l}\mathbf{C l} \% \\
\mathbf{w} / \mathbf{w}\end{array}$} & \multicolumn{4}{|c|}{ MDL (ng/g lipid) } \\
\hline & & & & & vSCCPs & SCCPs & MCCPs & LCCPs & & vSCCPs & SCCPs & MCCPs & LCCPs \\
\hline Narwhal & muscle & Melville, Greenland & 2018 & male & 1.00 & 31 & $<48$ & $<2.4$ & $51 \%$ & 0.61 & 30 & 48 & 2.4 \\
\hline Narwhal & muscle & Melville, Greenland & 2018 & male & 0.64 & $<22$ & $<35$ & $<1.7$ & $51 \%$ & 0.45 & 22 & 35 & 1.7 \\
\hline Narwhal & blubber & Melville, Greenland & 2018 & male & 1.10 & 16 & $<10$ & 0.66 & $64 \%$ & 0.13 & 6.4 & 10 & 0.5 \\
\hline Narwhal & blubber & Melville, Greenland & 2018 & male & 0.68 & $<5.2$ & $<8.3$ & $<0.41$ & $64 \%$ & 0.11 & 5.2 & 8.3 & 0.41 \\
\hline Harbor porpoise & blubber & Maniitsoq, Greenland & 2018 & - & 1.30 & 13 & $<18$ & $<0.87$ & $64 \%$ & 0.22 & 11 & 18 & 0.87 \\
\hline Harbor porpoise & blubber & Maniitsoq, Greenland & 2018 & large & $<0.12$ & $<6.1$ & $<9.8$ & $<0.48$ & $62 \%$ & 0.12 & 6.1 & 9.8 & 0.48 \\
\hline Harbor porpoise & blubber & Nuuk, Greenland & 2018 & 8 years & 0.63 & 11 & $<10$ & $<0.5$ & $60 \%$ & 0.13 & 6.3 & 10 & 0.5 \\
\hline Harbor porpoise & blubber & Nuuk, Greenland & 2018 & calf & 0.82 & 24 & 18 & 1.10 & $57 \%$ & 0.23 & 11 & 18 & 0.9 \\
\hline Harbor porpoise & blubber & Nuuk, Greenland & 2018 & large & 0.58 & 9.80 & $<15$ & $<0.76$ & $64 \%$ & 0.2 & 9.6 & 15 & 0.76 \\
\hline Blue mussel & soft tissue & Nuuk, Greenland & 2020 & (pooled) & 0.72 & 69 & 87 & 26 & $53 \%$ & 0.27 & 13 & 21 & 1.0 \\
\hline Blue mussel & soft tissue & Nuuk, Greenland & 2020 & (pooled) & 2.20 & 300 & 250 & 67 & $57 \%$ & 0.35 & 17 & 28 & 1.4 \\
\hline Iceland scallop & soft tissue & Nuuk, Greenland & 2018 & (pooled) & $<1.4$ & $<68$ & 120 & 10 & $52 \%$ & 1.4 & 68 & 110 & 5.3 \\
\hline Killer whale & muscle & Tasiilaq, Greenland & 2016 & female & 0.22 & 20 & 35 & 2.00 & $53 \%$ & 0.15 & 7.4 & 12 & 0.58 \\
\hline Killer whale & blubber & Tasiilaq, Greenland & 2016 & female & 0.21 & 9.70 & $<14$ & $<0.67$ & $57 \%$ & 0.17 & 8.5 & 14 & 0.67 \\
\hline Minke whale & muscle & Tasiilaq, Greenland & 2017 & fetus female & 0.14 & 6 & 19 & 0.73 & $52 \%$ & 0.1 & 4.9 & 7.9 & 0.39 \\
\hline Minke whale & muscle & Tasiilaq, Greenland & 2017 & adult female & 0.51 & 20 & 85 & 2.90 & $51 \%$ & 0.31 & 15 & 24 & 1.2 \\
\hline Pilot whale & muscle & Tasiilaq, Greenland & 2018 & calf female & $<0.24$ & 32 & 37 & 3.30 & $53 \%$ & 0.24 & 12 & 19 & 0.92 \\
\hline Pilot whale & muscle & Tasiilaq, Greenland & 2018 & adult male & $<0.13$ & 26 & 50 & 1.60 & $52 \%$ & 0.13 & 6.5 & 10 & 0.51 \\
\hline Pilot whale & muscle & Tasiilaq, Greenland & 2018 & adult female, pregnant & 0.20 & 8 & 20 & 0.61 & $53 \%$ & 0.12 & 5.9 & 9.5 & 0.47 \\
\hline Pilot whale & blubber & Tasiilaq, Greenland & 2018 & calf female & 0.16 & 8.30 & $<12$ & $<0.59$ & $66 \%$ & 0.15 & 7.5 & 12 & 0.59 \\
\hline Pilot whale & blubber & Tasiilaq, Greenland & 2018 & adult male & $<0.17$ & 27 & 17 & $<0.67$ & $52 \%$ & 0.17 & 8.5 & 14 & 0.67 \\
\hline Pilot whale & blubber & Tasiilaq, Greenland & 2018 & adult female, pregnant & 0.22 & $<5.4$ & $<8.6$ & $<0.42$ & $57 \%$ & 0.11 & 5.4 & 8.6 & 0.42 \\
\hline Greenland shark & liver & Waters around Iceland & 2001 & adult female & 1.40 & 15 & 5.10 & 0.92 & $56 \%$ & 0.054 & 2.6 & 4.2 & 0.21 \\
\hline Greenland shark & liver & Waters around Iceland & 2002 & adult female & 0.10 & 3.20 & 5.00 & 0.45 & $56 \%$ & 0.060 & 3.0 & 4.7 & 0.23 \\
\hline Killer whale & muscle & Mollösund, Sweden & 2018 & male, c.a. 6-meter length & 34 & 570 & 270 & 930 & $48 \%$ & 1.7 & 83 & 130 & 6.5 \\
\hline Killer whale & blubber & Mollösund, Sweden & 2018 & male, c.a. 6-meter length & 18 & 280 & 74 & 32 & $59 \%$ & 0.11 & 5.5 & 8.8 & 0.44 \\
\hline Harbor porpoise & blubber & Öresund, Sweden & 2016 & adult male & 2.8 & 79 & 18 & 11 & $63 \%$ & 0.12 & 6.0 & 1.5 & 0.62 \\
\hline Harbor porpoise & blubber & Öresund, Sweden & 2017 & adult female & 1.5 & 25 & 17 & 13 & $61 \%$ & 0.12 & 6.1 & 1.6 & 0.63 \\
\hline Harbor porpoise & blubber & Öresund, Sweden & 2018 & adult male & 3.2 & 110 & 14 & 12 & $64 \%$ & 0.12 & 6.2 & 1.6 & 0.64 \\
\hline
\end{tabular}


Table S2. Comparison of worldwide concentrations of CPs and other POPs in the same marine species as the current study.

\begin{tabular}{|c|c|c|c|c|c|c|c|}
\hline species & tissue & site & year & sample info & pollutants & median (range), ng/g lipid & reference \\
\hline humpback whales & blubber & Australian coastline & $2007-2015$ & both sex, 2 adults, 4 juveniles, and 3 calves & SCCPs & $20(<\mathrm{MDL}-46)$ & Casa et al. $(2019)^{11}$ \\
\hline beluga & blubber & NW Greenland & 1989 & 3 females, 1 male & SCCPs & $220(110-250)$ & Tomy et al. $(2000)^{12}$ \\
\hline harbor porpoises & blubber & Baltic Sea & 2008 & 1 pool of adults, 3 females and 1 male & SCCPs & 300 & de Wit et al. $(2020)^{13}$ \\
\hline harbor porpoises & blubber & Baltic Sea & 2008 & 1 pool of adults, 3 females and 1 male & MCCPs & 36 & de Wit et al. $(2020)^{13}$ \\
\hline harbor porpoises & blubber & Baltic Sea & 2008 & 1 pool of adults, 3 females and 1 male & LCCPs & 48 & de Wit et al. $(2020)^{13}$ \\
\hline harbor porpoises & blubber & Baltic Sea & 2006-2012 & 1 pool of adults, 1 female and 1 male & SCCPs & 120 & de Wit et al. $(2020)^{13}$ \\
\hline harbor porpoises & blubber & Baltic Sea & 2006-2012 & 1 pool of adults, 1 female and 1 male & MCCPs & 59 & de Wit et al. $(2020)^{13}$ \\
\hline harbor porpoises & blubber & Baltic Sea & 2006-2012 & 1 pool of adults, 1 female and 1 male & LCCPs & 25 & de Wit et al. $(2020)^{13}$ \\
\hline scallop & soft tissue & Bohai Sea, China & 2009 & 7 pooled samples & SCCPs & $2600(950-17000)$ & Yuan et al. $(2012)^{14}$ \\
\hline blue mussel & soft tissue & Bohai Sea, China & 2009 & 5 pooled samples & SCCPs & $2400(1400-24000)$ & Yuan et al. $(2012)^{14}$ \\
\hline blue mussel & soft tissue & Ijmuiden harbor, the Netherlands & 2013 & 1 pooled sample & SCCPs & 900 & Yuan et al. $(2018)^{15}$ \\
\hline blue mussel & soft tissue & Ijmuiden harbor, the Netherlands & 2013 & 1 pooled sample & MCCPs & 1500 & Yuan et al. $(2018)^{15}$ \\
\hline blue mussel & soft tissue & Ijmuiden harbor, the Netherlands & 2013 & 1 pooled sample & LCCPs & 440 & Yuan et al. $(2018)^{15}$ \\
\hline blue mussel & soft tissue & Chile & 2016 & 1 pooled sample & SCCPs & 770 & Yuan et al. $(2018)^{15}$ \\
\hline blue mussel & soft tissue & Chile & 2016 & 1 pooled sample & MCCPs & 2200 & Yuan et al. $(2018)^{15}$ \\
\hline blue mussel & soft tissue & Chile & 2016 & 1 pooled sample & LCCPs & 650 & Yuan et al. $(2018)^{15}$ \\
\hline blue mussel & soft tissue & Baltic Sea & 2015 & 1 pooled sample & $\Sigma \mathrm{CPs}$ & 400 & de Wit et al. $(2020)^{13}$ \\
\hline blue mussel & soft tissue & Baltic Sea & 2006 & 1 pooled sample & $\Sigma \mathrm{PCBs}$ & 350 & de Wit et al. $(2020)^{13}$ \\
\hline blue mussel & soft tissue & Baltic Sea & 2006 & 1 pooled sample & $\Sigma$ DDT & 78 & de Wit et al. $(2020)^{13}$ \\
\hline blue mussel & soft tissue & Baltic Sea & 2015 & 1 pooled sample & $\Sigma$ PBDEs & 1.8 & de Wit et al. $(2020)^{13}$ \\
\hline killer whale & blubber & Tasiilaq, Greenland & $2008-2014$ & 6 adult females & $\Sigma$ PCBs & $48600(19600-65500)$ & Pedro et al. $(2017)^{16}$ \\
\hline killer whale & blubber & Tasiilaq, Greenland & 2012 & 2 fetuses & $\Sigma \mathrm{PCBs}$ & $11400(9470-13400)$ & Pedro et al. $(2017)^{16}$ \\
\hline killer whale & blubber & Esbjerg, Denmark & 2005 & 1 male & $\Sigma \mathrm{PCBs}$ & 96100 & Pedro et al. $(2017)^{16}$ \\
\hline killer whale & blubber & Tasiilaq, Greenland & $2008-2014$ & 6 adult females & $\Sigma \mathrm{HCH}$ & $68.2(31.0-103)$ & Pedro et al. $(2017)^{16}$ \\
\hline killer whale & blubber & Tasiilaq, Greenland & 2012 & 2 fetuses & $\Sigma \mathrm{HCH}$ & $53.1(29.0-77.1)$ & Pedro et al. $(2017)^{16}$ \\
\hline killer whale & blubber & Esbjerg, Denmark & 2005 & 1 male & $\Sigma \mathrm{HCH}$ & 87.2 & Pedro et al. $(2017)^{16}$ \\
\hline killer whale & blubber & Tasiilaq, Greenland & $2008-2014$ & 6 adult females & $\Sigma$ DDT & $30100(16100-50200)$ & Pedro et al. $(2017)^{16}$ \\
\hline killer whale & blubber & Tasiilaq, Greenland & 2012 & 2 fetuses & $\Sigma$ DDT & $13400(7890-18900)$ & Pedro et al. $(2017)^{16}$ \\
\hline killer whale & blubber & Esbjerg, Denmark & 2005 & 1 male & $\Sigma$ DDT & 85400 & Pedro et al. $(2017)^{16}$ \\
\hline killer whale & blubber & northern Norway & 2015-2017 & both sexes, $n=8$ & $\Sigma \mathrm{PCBs}$ & $15000(6500-100000)$ & Andvik et al. $(2021)^{17}$ \\
\hline
\end{tabular}




\begin{tabular}{|c|c|c|c|c|c|c|c|}
\hline killer whale & blubber & northern Norway & 2015-2017 & both sexes, $n=8$ & $\Sigma \mathrm{DDT}$ & $7000(3900-82000)$ & Andvik et al. $(2021)^{17}$ \\
\hline killer whale & blubber & northern Norway & $2015-2017$ & both sexes, $n=8$ & $\Sigma \mathrm{HCH}$ & $65(4.4-180)$ & Andvik et al. $(2021)^{17}$ \\
\hline killer whale & blubber & northern Norway & 2015-2017 & both sexes, $n=8$ & $\Sigma$ PBDEs & $350(170-1700)$ & Andvik et al. $(2021)^{17}$ \\
\hline killer whale & muscle & northern Norway & $2015-2017$ & both sexes, $n=8$ & $\Sigma \mathrm{PCBs}$ & $33000(11000-100000)$ & Andvik et al. $(2021)^{17}$ \\
\hline killer whale & muscle & northern Norway & $2015-2017$ & both sexes, $n=8$ & $\Sigma$ DDT & $13000(6400-75000)$ & Andvik et al. $(2021)^{17}$ \\
\hline killer whale & muscle & northern Norway & $2015-2017$ & both sexes, $n=8$ & $\Sigma \mathrm{HCH}$ & $50(26-110)$ & Andvik et al. $(2021)^{17}$ \\
\hline killer whale & muscle & northern Norway & $2015-2017$ & both sexes, $n=8$ & $\Sigma$ PBDEs & $860(210-2500)$ & Andvik et al. $(2021)^{17}$ \\
\hline harbor porpoises & blubber & west coast, Sweden & 1996 & 3-7 y male, $n=3$ & $\Sigma \mathrm{PCBs}$ & $12000(6300-21000)$ & K. Karlson et al. $(2000)^{18}$ \\
\hline harbor porpoises & nuchal & west coast, Sweden & 1996 & $3-7$ y male, $n=3$ & $\Sigma \mathrm{PCBs}$ & $8100(7900-26000)$ & K. Karlson et al. $(2000)^{18}$ \\
\hline harbor porpoises & liver & west coast, Sweden & 1996 & 3-7 y male, $n=3$ & $\Sigma \mathrm{PCBs}$ & $10000(5700-24000)$ & K. Karlson et al. $(2000)^{18}$ \\
\hline harbor porpoises & muscle & west coast, Sweden & 1996 & $3-7$ y male, $n=3$ & $\Sigma \mathrm{PCBs}$ & $7000(5800-15000)$ & K. Karlson et al. $(2000)^{18}$ \\
\hline harbor porpoises & brain & west coast, Sweden & 1996 & 3-7 y male, $n=3$ & $\Sigma \mathrm{PCBs}$ & $3600(840-4200)$ & K. Karlson et al. $(2000)^{18}$ \\
\hline harbor porpoises & blubber & west coast, Sweden & 1996 & 3-7 y male, $n=3$ & $\Sigma$ DDT & $2300(1900-4800)$ & K. Karlson et al. $(2000)^{18}$ \\
\hline harbor porpoises & nuchal & west coast, Sweden & 1996 & $3-7$ y male, $n=3$ & $\Sigma$ DDT & $2100(1800-4900)$ & K. Karlson et al. $(2000)^{18}$ \\
\hline harbor porpoises & liver & west coast, Sweden & 1996 & $3-7$ y male, $n=3$ & $\Sigma$ DDT & $1800(1300-4500)$ & K. Karlson et al. $(2000)^{18}$ \\
\hline harbor porpoises & muscle & west coast, Sweden & 1996 & $3-7$ y male, $n=3$ & $\Sigma$ DDT & $1200(910-3000)$ & K. Karlson et al. $(2000)^{18}$ \\
\hline harbor porpoises & brain & west coast, Sweden & 1996 & $3-7$ y male, $n=3$ & $\Sigma$ DDT & $250(170-690)$ & K. Karlson et al. $(2000)^{18}$ \\
\hline harbor porpoises & blubber & West Iceland & 1997 & 3 pooled samples, 5 male individuals/pool & $\Sigma$ PBDEs & $75(67-96)$ & Rotander et al. $(2012)^{19}$ \\
\hline harbor porpoises & blubber & Baltic Sea & 2008 & 1 pooled sample, juvenile male and female & $\Sigma$ PBDEs & 210 & de Wit et al. $(2020)^{13}$ \\
\hline harbor porpoises & blubber & Baltic Sea & 2006-2012 & 1 pooled sample, adult females, males & $\Sigma$ PBDEs & 230 & de Wit et al. $(2020)^{13}$ \\
\hline harbor porpoises & blubber & Baltic Sea & 2008 & 1 pooled sample, juvenile male and female & $\Sigma$ PCBs & 4500 & de Wit et al. $(2020)^{13}$ \\
\hline harbor porpoises & blubber & Baltic Sea & 2006-2012 & 1 pooled sample, adult females, males & $\Sigma \mathrm{PCBs}$ & 5600 & de Wit et al. $(2020)^{13}$ \\
\hline harbor porpoises & blubber & Baltic Sea & 2008 & 1 pooled sample, juvenile male and female & $\Sigma$ DDT & 2800 & de Wit et al. $(2020)^{13}$ \\
\hline harbor porpoises & blubber & Baltic Sea & 2006-2012 & 1 pooled sample, adult females, males & $\Sigma$ DDT & 3900 & de Wit et al. $(2020)^{13}$ \\
\hline blue mussel & soft tissue & Usuk, Greenland & 2000 & 1 pooled sample, $5(4-6) \mathrm{cm}$ & BDE-47 & 5 & Christensen et al. $(2002)^{20}$ \\
\hline blue mussel & soft tissue & Usuk, Greenland & 2000 & 1 pooled sample, $5(4-6) \mathrm{cm}$ & BDE-99 & 1 & Christensen et al. $(2002)^{20}$ \\
\hline blue mussel & soft tissue & Usuk, Greenland & 2000 & 1 pooled sample, $5(4-6) \mathrm{cm}$ & BDE-100 & $<1$ & Christensen et al. $(2002)^{20}$ \\
\hline blue mussel & soft tissue & Usuk, Greenland & 2000 & 1 pooled sample, $5(4-6) \mathrm{cm}$ & BDE-153 & $<2.5$ & Christensen et al. $(2002)^{20}$ \\
\hline blue mussel & soft tissue & Usuk, Greenland & 2000 & 1 pooled sample, $5(4-6) \mathrm{cm}$ & $\Sigma$ PBDEs & 5.5 & Christensen et al. $(2002)^{20}$ \\
\hline blue mussel & soft tissue & Usuk, Greenland & 2000 & 1 pooled sample, $5(4-6) \mathrm{cm}$ & $\Sigma \mathrm{PCBs}$ & 38 & Christensen et al. $(2002)^{20}$ \\
\hline blue mussel & soft tissue & Iceland & $1993-2010$ & pooled samples from 11 subsites, $n=11$ & PCB-153 & $33(7.8-64)$ & Sturludottir et al. $(2013)^{21}$ \\
\hline pilot whale & liver & Faroe Islands & $2010 / 2011$ & 10 adult females/ 2 adult males $/ 80-2 \mathrm{yr}$ juv & $\Sigma \mathrm{PCBs}$ & $2523 / 16678 / 14364$ & Hoydal et al. $(2015)^{22}$ \\
\hline pilot whale & liver & Faroe Islands & $2010 / 2011$ & 10 adult females/2 adult males/8 0-2 yr juv & pp-DDE & $1227 / 14240 / 8549$ & Hoydal et al. $(2015)^{22}$ \\
\hline
\end{tabular}




\begin{tabular}{|c|c|c|c|c|c|c|c|}
\hline pilot whale & liver & Faroe Islands & $2010 / 2011$ & 10 adult females $/ 2$ adult males $/ 80-2$ yr juv & $\Sigma$ PBDEs & $128.5 / 460.3 / 725.7$ & Hoydal et al. $(2015)^{22}$ \\
\hline pilot whale & blubber & Faroe Islands & $2006 / 2007$ & 3 pooled samples, $3-5$ male individuals/pool & $\Sigma$ PBDEs & $1041(840-1307)$ & Rotander et al. $(2012)^{19}$ \\
\hline pilot whale & blubber & Faroe Islands & 2013 & 5 juvenile males & BDE-28 & $10(7.6-58)$ & Bjurlid et al. $(2018)^{23}$ \\
\hline pilot whale & blubber & Faroe Islands & 2013 & 5 juvenile males & BDE-47 & $220(150-1200)$ & Bjurlid et al. $(2018)^{23}$ \\
\hline pilot whale & blubber & Faroe Islands & 2013 & 5 juvenile males & BDE-66 & $7.2(5.4-21)$ & Bjurlid et al. $(2018)^{23}$ \\
\hline pilot whale & blubber & Faroe Islands & 2013 & 5 juvenile males & BDE-100 & $44(32-200)$ & Bjurlid et al. $(2018)^{23}$ \\
\hline pilot whale & blubber & Faroe Islands & 2013 & 5 juvenile males & BDE-99 & $42(31-240)$ & Bjurlid et al. $(2018)^{23}$ \\
\hline pilot whale & blubber & Faroe Islands & 2013 & 5 juvenile males & BDE-85 & $1.7(1.3-4.2)$ & Bjurlid et al. $(2018)^{23}$ \\
\hline pilot whale & blubber & Faroe Islands & 2013 & 5 juvenile males & BDE-154 & $41(30-180)$ & Bjurlid et al. $(2018)^{23}$ \\
\hline pilot whale & blubber & Faroe Islands & 2013 & 5 juvenile males & BDE-153 & $13(9.2-59)$ & Bjurlid et al. $(2018)^{23}$ \\
\hline pilot whale & blubber & Faroe Islands & 2013 & 5 juvenile males & BDE-183 & $0.40(0.40-1.4)$ & Bjurlid et al. $(2018)^{23}$ \\
\hline pilot whale & blubber & Faroe Islands & 2013 & 5 juvenile males & $\Sigma$ PBDEs & $380(270-1900)$ & Bjurlid et al. $(2018)^{23}$ \\
\hline minke whale & blubber & West Greenland & 1998 & 3 pooled samples, 4 female individuals/pool & इPBDEs & $71(50-170)$ & Rotander et al. $(2012)^{19}$ \\
\hline minke whale & blubber & Norway arctic & 1999 & - & BDE-154 & $6.46(2.93-24.89)$ & Norden $(2011)^{24}$ \\
\hline minke whale & blubber & Norway arctic & 1999 & - & BDE-209 & not detected & Norden $(2011)^{24}$ \\
\hline minke whale & blubber & West Greenland & 1998 & - & BDE-28 & $1.4(0.7-3.1)$ & Norden $(2011)^{24}$ \\
\hline minke whale & blubber & West Greenland & 1998 & - & BDE-47 & $68(41-69)$ & Norden $(2011)^{24}$ \\
\hline minke whale & blubber & West Greenland & 1998 & - & BDE-66 & $0.5(0.1-3.6)$ & Norden $(2011)^{24}$ \\
\hline minke whale & blubber & West Greenland & 1998 & - & BDE-100 & $7.3(3.9-14)$ & Norden $(2011)^{24}$ \\
\hline minke whale & blubber & West Greenland & 1998 & - & BDE-99 & $12(7.1-63)$ & Norden $(2011)^{24}$ \\
\hline minke whale & blubber & West Greenland & 1998 & - & BDE-85 & $2.5(0.4-2.7)$ & Norden $(2011)^{24}$ \\
\hline minke whale & blubber & West Greenland & 1998 & - & BDE-154 & $7(6.3-10)$ & Norden $(2011)^{24}$ \\
\hline minke whale & blubber & West Greenland & 1998 & - & BDE-153 & $1.5(1.5-5.4)$ & Norden $(2011)^{24}$ \\
\hline minke whale & blubber & West Greenland & 1998 & - & BDE-138 & $0.05(<0.1-0.1)$ & Norden $(2011)^{24}$ \\
\hline minke whale & blubber & West Greenland & 1998 & - & BDE-183 & $0.1(0.1-0.2)$ & Norden $(2011)^{24}$ \\
\hline minke whale & blubber & West Greenland & 1998 & - & ¿PBDEs & $99(64-170)$ & Norden $(2011)^{24}$ \\
\hline Greenland shark & liver & Iceland & 2001-2003 & 10 females & $\Sigma \mathrm{PCBs}$ & $4400(990-10000)$ & Strid et al. $(2007)^{25}$ \\
\hline Greenland shark & liver & Iceland & $2001-2003$ & 10 females & $\Sigma$ PBDEs & $41(9.9-200)$ & Strid et al. $(2010)^{26}$ \\
\hline Greenland shark & liver & Cumberland Sound, Canada & 1999 & 5 females, 10 males & $\Sigma$ PCBs & $3442 *$ & Fisk et al. $(2002)^{27}$ \\
\hline Greenland shark & liver & Cumberland Sound, Canada & 1999 & 5 females, 10 males & $\Sigma$ DDT & $7159^{*}$ & Fisk et al. $(2002)^{27}$ \\
\hline Greenland shark & liver & Greenland & 2012-2014 & 9 female, 6 males & $\Sigma$ DDT & 523 (na-1444) & Cotronei et al. $(2018)^{28}$ \\
\hline
\end{tabular}


Table S3. Relative contributions of chlorinated paraffin standards in pattern reconstruction of individual samples.

\begin{tabular}{|c|c|c|c|c|c|c|c|c|c|c|c|c|c|c|c|c|c|c|c|c|}
\hline \multirow[b]{2}{*}{ Sample } & \multirow[b]{2}{*}{ Year } & \multirow[b]{2}{*}{$\begin{array}{c}\operatorname{map} \\
i^{\mathrm{a}}\end{array}$} & \multirow[b]{2}{*}{$R^{2}$} & \multicolumn{5}{|c|}{$\begin{array}{l}\text { Relative contributions from } \\
\text { SCCP products }\end{array}$} & \multicolumn{7}{|c|}{$\begin{array}{c}\text { Relative contributions from } \\
\text { MCCP products }\end{array}$} & \multicolumn{5}{|c|}{$\begin{array}{c}\text { Relative contributions from } \\
\text { LCCP products }\end{array}$} \\
\hline & & & & 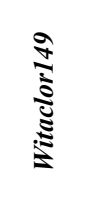 & 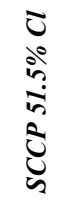 & 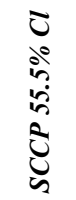 & $\begin{array}{l}\tilde{0} \\
\dot{0} \\
\dot{6} \\
\tilde{U} \\
0\end{array}$ & 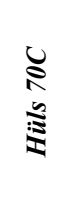 & 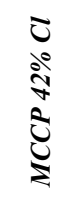 & 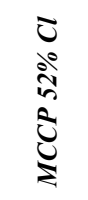 & 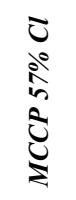 & 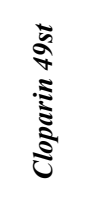 & 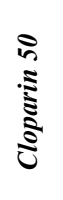 & 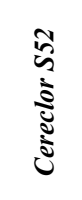 & 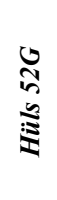 & 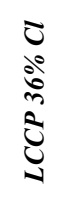 & 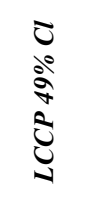 & 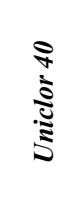 & 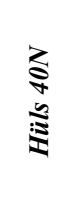 & 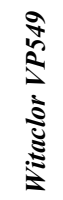 \\
\hline Narwhal & 2018 & 1 & 0.97 & $90 \%$ & & $4 \%$ & $5 \%$ & $1 \%$ & & & & & & & & & & & & \\
\hline Narwhal & 2018 & 1 & $-b$ & & & & & & & & & & & & & & & & & \\
\hline Narwhal & 2018 & 1 & 0.35 & $9 \%$ & & & $83 \%$ & $5 \%$ & & & & & & & & $3 \%$ & & & & \\
\hline Narwhal & 2018 & 1 & - & & & & & & & & & & & & & & & & & \\
\hline Harbor porpoise & 2018 & 2 & 0.57 & $11 \%$ & $0 \%$ & $2 \%$ & $83 \%$ & $3 \%$ & & & & & & & & & & & & \\
\hline Harbor porpoise & 2018 & 2 & - & & & & & & & & & & & & & & & & & \\
\hline Harbor porpoise & 2018 & 3 & 0.58 & & & $26 \%$ & $70 \%$ & $3 \%$ & & & & & & & & & & & & \\
\hline Harbor porpoise & 2018 & 3 & 0.76 & $10 \%$ & $0 \%$ & $0 \%$ & $1 \%$ & $0 \%$ & $83 \%$ & & $0 \%$ & & & & & $0 \%$ & $6 \%$ & & & \\
\hline Harbor porpoise & 2018 & 3 & 0.52 & $11 \%$ & $0 \%$ & $2 \%$ & $83 \%$ & $3 \%$ & & & & & & & & & & & & \\
\hline Blue mussel & 2020 & 3 & 0.88 & & $2 \%$ & $0 \%$ & $0 \%$ & $0 \%$ & & $0 \%$ & $0 \%$ & $0 \%$ & & $87 \%$ & & $0 \%$ & $11 \%$ & & & $0 \%$ \\
\hline Blue mussel & 2020 & 3 & 0.66 & $12 \%$ & & $3 \%$ & $5 \%$ & $1 \%$ & $27 \%$ & $34 \%$ & $0 \%$ & $1 \%$ & & & & $1 \%$ & $13 \%$ & & & $4 \%$ \\
\hline Iceland scallop & 2018 & 3 & 0.96 & & & & & & $67 \%$ & $31 \%$ & $0 \%$ & $0 \%$ & & $0 \%$ & & $0 \%$ & $1 \%$ & $1 \%$ & & $1 \%$ \\
\hline Killer whale & 2016 & 4 & 0.96 & $11 \%$ & & & $3 \%$ & $2 \%$ & $46 \%$ & $11 \%$ & $1 \%$ & $13 \%$ & & $6 \%$ & & $0 \%$ & $7 \%$ & $0 \%$ & & \\
\hline Killer whale & 2016 & 4 & 0.82 & & & $2 \%$ & $12 \%$ & $1 \%$ & $58 \%$ & $24 \%$ & $0 \%$ & $1 \%$ & & $0 \%$ & & $1 \%$ & $2 \%$ & $0 \%$ & $0 \%$ & \\
\hline Minke whale & 2017 & 4 & 0.96 & $7 \%$ & & $0 \%$ & $0 \%$ & $0 \%$ & $34 \%$ & & $0 \%$ & $0 \%$ & & $55 \%$ & & $0 \%$ & $4 \%$ & $0 \%$ & $0 \%$ & $0 \%$ \\
\hline Minke whale & 2017 & 4 & 0.97 & $7 \%$ & & $0 \%$ & $0 \%$ & $0 \%$ & $74 \%$ & $16 \%$ & $0 \%$ & $0 \%$ & & & & $0 \%$ & $2 \%$ & $0 \%$ & $0 \%$ & \\
\hline Pilot whale & 2018 & 4 & 0.76 & $20 \%$ & & $0 \%$ & $0 \%$ & $0 \%$ & $31 \%$ & & $0 \%$ & $0 \%$ & & $34 \%$ & & $0 \%$ & $15 \%$ & & & \\
\hline Pilot whale & 2018 & 4 & 0.98 & $12 \%$ & & $0 \%$ & $0 \%$ & $0 \%$ & $60 \%$ & $22 \%$ & $0 \%$ & $0 \%$ & & & & $0 \%$ & $5 \%$ & & & \\
\hline Pilot whale & 2018 & 4 & 0.93 & $6 \%$ & $0 \%$ & $0 \%$ & $0 \%$ & $0 \%$ & $44 \%$ & $1 \%$ & $0 \%$ & $0 \%$ & & $45 \%$ & & $0 \%$ & $4 \%$ & $0 \%$ & $0 \%$ & $0 \%$ \\
\hline Pilot whale & 2018 & 4 & 0.34 & $90 \%$ & & $3 \%$ & $7 \%$ & $0 \%$ & & & & & & & & & & & & \\
\hline Pilot whale & 2018 & 4 & 0.98 & $13 \%$ & & $0 \%$ & $0 \%$ & $0 \%$ & $63 \%$ & $23 \%$ & $0 \%$ & $0 \%$ & & & & & & & & \\
\hline Pilot whale & 2018 & 4 & - & & & & & & & & & & & & & & & & & \\
\hline Greenland shark & 2001 & 5 & 0.61 & $40 \%$ & & $0 \%$ & $0 \%$ & $0 \%$ & $28 \%$ & $2 \%$ & $0 \%$ & $0 \%$ & & $26 \%$ & & $0 \%$ & $3 \%$ & & & $0 \%$ \\
\hline Greenland shark & 2002 & 5 & 0.64 & $2 \%$ & $4 \%$ & $0 \%$ & $0 \%$ & $0 \%$ & & $1 \%$ & $0 \%$ & $0 \%$ & & $89 \%$ & & $0 \%$ & $4 \%$ & & $0 \%$ & $0 \%$ \\
\hline Killer whale & 2018 & 6 & 0.82 & $11 \%$ & $0 \%$ & $1 \%$ & $4 \%$ & $0 \%$ & $58 \%$ & $1 \%$ & $0 \%$ & & & & & $0 \%$ & $0 \%$ & $24 \%$ & & $1 \%$ \\
\hline Killer whale & 2018 & 6 & 0.60 & $21 \%$ & $0 \%$ & $0 \%$ & $0 \%$ & $0 \%$ & $76 \%$ & $1 \%$ & $0 \%$ & $0 \%$ & & & & $0 \%$ & & $1 \%$ & $0 \%$ & $0 \%$ \\
\hline Harbor porpoise & 2016 & 7 & 0.59 & & & & $61 \%$ & $2 \%$ & & $1 \%$ & $35 \%$ & & & & & & $1 \%$ & $0 \%$ & & $0 \%$ \\
\hline Harbor porpoise & 2017 & 7 & 0.72 & & & & $32 \%$ & $8 \%$ & & & $48 \%$ & $2 \%$ & & & & $3 \%$ & $4 \%$ & $3 \%$ & & $1 \%$ \\
\hline Harbor porpoise & 2018 & 7 & 0.61 & & & & $74 \%$ & $1 \%$ & $0 \%$ & $2 \%$ & $22 \%$ & & & & & & $0 \%$ & $1 \%$ & & \\
\hline
\end{tabular}


Table S4. Goodness of fit $\left(\mathrm{R}^{2}\right)$ between homologue profile of vSCCPs in CP-52 and the profile in individual samples.

\begin{tabular}{|c|c|c|c|}
\hline Sample & Year & $\operatorname{map} i^{\text {a }}$ & $R^{2}$ \\
\hline Narwhal & 2018 & 1 & 0.57 \\
\hline Narwhal & 2018 & 1 & 0.37 \\
\hline Narwhal & 2018 & 1 & 0.64 \\
\hline Narwhal & 2018 & 1 & 0.63 \\
\hline Harbor porpoise & 2018 & 2 & 0.47 \\
\hline Harbor porpoise & 2018 & 2 & $-b$ \\
\hline Harbor porpoise & 2018 & 3 & 0.49 \\
\hline Harbor porpoise & 2018 & 3 & 0.54 \\
\hline Harbor porpoise & 2018 & 3 & 0.51 \\
\hline Blue mussel & 2020 & 3 & 0.73 \\
\hline Blue mussel & 2020 & 3 & 0.14 \\
\hline Iceland scallop & 2018 & 3 & $-\mathrm{b}$ \\
\hline Killer whale & 2016 & 4 & 0.51 \\
\hline Killer whale & 2016 & 4 & 0.52 \\
\hline Minke whale & 2017 & 4 & 0.51 \\
\hline Minke whale & 2017 & 4 & 0.61 \\
\hline Pilot whale & 2018 & 4 & $-\mathrm{b}$ \\
\hline Pilot whale & 2018 & 4 & $-{ }^{b}$ \\
\hline Pilot whale & 2018 & 4 & 0.44 \\
\hline Pilot whale & 2018 & 4 & 0.54 \\
\hline Pilot whale & 2018 & 4 & $-\mathrm{b}$ \\
\hline Pilot whale & 2018 & 4 & 0.45 \\
\hline Greenland shark & 2001 & 5 & 0.80 \\
\hline Greenland shark & 2002 & 5 & 0.59 \\
\hline Killer whale & 2018 & 6 & 0.83 \\
\hline Killer whale & 2018 & 6 & 0.57 \\
\hline Harbor porpoise & 2016 & 7 & 0.40 \\
\hline Harbor porpoise & 2017 & 7 & 0.60 \\
\hline Harbor porpoise & 2018 & 7 & 0.40 \\
\hline
\end{tabular}

a. see Figure 1; b. below MDL. 


\section{Reference}

1. Bogdal, C.; Alsberg, T.; Diefenbacher, P. S.; MacLeod, M.; Berger, U., Fast quantification of chlorinated paraffins in environmental samples by direct injection high-resolution mass spectrometry with pattern deconvolution. Anal. Chem. 2015, 87 (5), 2852-60.

2. Du, X.; Yuan, B.; Zhou, Y.; de Wit, C. A.; Zheng, Z.; Yin, G., Chlorinated Paraffins in Two Snake Species from the Yangtze River Delta: Tissue Distribution and Biomagnification. Environ. Sci. Technol. 2020, 54 (5), 2753-2762.

3. Du, X.; Yuan, B.; Zhou, Y.; Zheng, Z.; Wu, Y.; Qiu, Y.; Zhao, J.; Yin, G., Tissue-Specific Accumulation, Sexual Difference, and Maternal Transfer of Chlorinated Paraffins in Black-Spotted Frogs. Environ. Sci. Technol. 2019, 53 (9), 4739-4746.

4. Schinkel, L.; Bogdal, C.; Canonica, E.; Cariou, R.; Bleiner, D.; McNeill, K.; Heeb, N. V., Analysis of Medium-Chain and Long-Chain Chlorinated Paraffins: The Urgent Need for More Specific Analytical Standards. Environ. Sci. Technol. Lett. 2018, 5 (12), 708-717.

5. Mézière, M.; Krätschmer, K.; Perkons, I.; Zacs, D.; Marchand, P.; Dervilly, G.; Le Bizec, B.; Schächtele, A.; Cariou, R.; Vetter, W., Addressing Main Challenges Regarding Short- and MediumChain Chlorinated Paraffin Analysis Using GC/ECNI-MS and LC/ESI-MS Methods. J. Am. Soc. Mass Spectrom. 2020, 31 (9), 1885-1895.

6. Yuan, B.; Tay, J. H.; Papadopoulou, E.; Haug, L. S.; Padilla-Sánchez, J. A.; de Wit, C. A., Complex Mixtures of Chlorinated Paraffins Found in Hand Wipes of a Norwegian Cohort. Environ. Sci. Technol. Lett. 2020, 7 (3), 198-205.

7. Brits, M.; de Boer, J.; Rohwer, E. R.; De Vos, J.; Weiss, J. M.; Brandsma, S. H., Short-, medium-, and long-chain chlorinated paraffins in South African indoor dust and cat hair. Chemosphere 2020, 238, 124643 .

8. Heide-Jørgensen, M. P.; Dietz, R.; Laidre, K. L.; Richard, P.; Orr, J.; Schmidt, H. C., The migratory behaviour of narwhals (Monodon monoceros). Canadian Journal of Zoology 2003, 81 (8), 1298-1305.

9. Teilmann, J.; Christiansen, C. T.; Kjellerup, S.; Dietz, R.; Nachman, G., Geographic, seasonal, and diurnal surface behavior of harbor porpoises. Marine Mammal Science 2013, 29 (2), E60-E76.

10. Nielsen, N. H.; Teilmann, J.; Sveegaard, S.; Hansen, R. G.; Sinding, M. H. S.; Dietz, R.; HeideJørgensen, M. P., Oceanic movements, site fidelity and deep diving in harbour porpoises from Greenland show limited similarities to animals from the North Sea. Marine Ecology Progress Series 2018, 597, 259-272.

11. Casà, M. V.; van Mourik, L. M.; Weijs, L.; Mueller, J.; Nash, S. B., First detection of short-chain chlorinated paraffins (SCCPs) in humpback whales (Megaptera novaeangliae) foraging in Antarctic waters. Environmental Pollution 2019, 250, 953-959.

12. Tomy, G. T.; Muir, D. C. G.; Stern, G. A.; Westmore, J. B., Levels of C-10-C-13 polychloro-nalkanes in marine mammals from the Arctic and the St. Lawrence River estuary. Environmental Science \& Technology 2000, 34 (9), 1615-1619.

13. de Wit, C. A.; Bossi, R.; Dietz, R.; Dreyer, A.; Faxneld, S.; Garbus, S. E.; Hellström, P.; Koschorreck, J.; Lohmann, N.; Roos, A.; Sellström, U.; Sonne, C.; Treu, G.; Vorkamp, K.; Yuan, B.; Eulaers, I., Organohalogen compounds of emerging concern in Baltic Sea biota: Levels, biomagnification potential and comparisons with legacy contaminants. Environ. Int. 2020, 144, 106037.

14. Yuan, B.; Wang, T.; Zhu, N. L.; Zhang, K. G.; Zeng, L. X.; Fu, J. J.; Wang, Y. W.; Jiang, G. B., Short Chain Chlorinated Paraffins in Mollusks from Coastal Waters in the Chinese Bohai Sea. Environmental Science \& Technology 2012, 46 (12), 6489-6496.

15. Yuan, B.; Benskin, J. P.; Chen, C.-E. L.; Bergman, Å., Determination of Chlorinated Paraffins by Bromide-Anion Attachment Atmospheric-Pressure Chemical Ionization Mass Spectrometry. Environ. Sci. Technol. Lett. 2018, 5 (6), 348-353. 
16. Pedro, S.; Boba, C.; Dietz, R.; Sonne, C.; Rosing-Asvid, A.; Hansen, M.; Provatas, A.; McKinney, M. A., Blubber-depth distribution and bioaccumulation of PCBs and organochlorine pesticides in Arctic-invading killer whales. Science of The Total Environment 2017, 601-602, 237-246.

17. Andvik, C.; Jourdain, E.; Lyche, J. L.; Karoliussen, R.; Borgå, K., High Levels of Legacy and Emerging Contaminants in Killer Whales (Orcinus orca) from Norway, 2015 to 2017. Environmental Toxicology and Chemistry n/a (n/a).

18. Karlson, K.; Ishaq, R.; Becker, G.; Berggren, P.; Broman, D.; Colmsjö, A., PCBs, DDTs and methyl sulphone metabolites in various tissues of harbour porpoises from Swedish waters. Environmental Pollution 2000, 110 (1), 29-46.

19. Rotander, A.; van Bavel, B.; Polder, A.; Rigét, F.; Auðunsson, G. A.; Gabrielsen, G. W.; Víkingsson, G.; Bloch, D.; Dam, M., Polybrominated diphenyl ethers (PBDEs) in marine mammals from Arctic and North Atlantic regions, 1986-2009. Environment International 2012, 40, 102-109.

20. Christensen, J. H.; Glasius, M.; Pécseli, M.; Platz, J.; Pritzl, G., Polybrominated diphenyl ethers (PBDEs) in marine fish and blue mussels from southern Greenland. Chemosphere 2002, 47 (6), 631638.

21. Sturludottir, E.; Gunnlaugsdottir, H.; Jorundsdottir, H. O.; Magnusdottir, E. V.; Olafsdottir, K.; Stefansson, G., Spatial and temporal trends of contaminants in mussel sampled around the Icelandic coastline. Science of The Total Environment 2013, 454-455, 500-509.

22. Hoydal, K. S.; Letcher, R. J.; Blair, D. A. D.; Dam, M.; Lockyer, C.; Jenssen, B. M., Legacy and emerging organic pollutants in liver and plasma of long-finned pilot whales (Globicephala melas) from waters surrounding the Faroe Islands. Science of The Total Environment 2015, 520, 270-285.

23. Bjurlid, F.; Dam, M.; Hoydal, K.; Hagberg, J., Occurrence of polybrominated dibenzo-p-dioxins, dibenzofurans (PBDD/Fs) and polybrominated diphenyl ethers (PBDEs) in pilot whales (Globicephala melas) caught around the Faroe Islands. Chemosphere 2018, 195, 11-20.

24. Dam, M., New POPs in Marine Mammals in Nordic Arctic and NE Atlantic Areas during Three Decades. Nordic Council of Ministers: 2011.

25. Strid, A.; Jörundsdóttir, H.; Päpke, O.; Svavarsson, J.; Bergman, Å., Dioxins and PCBs in Greenland shark (Somniosus microcephalus) from the North-East Atlantic. Marine Pollution Bulletin 2007, 54 (9), 1514-1522.

26. Strid, A.; Athanassiadis, I.; Athanasiadou, M.; Svavarsson, J.; Päpke, O.; Bergman, Å., Neutral and phenolic brominated organic compounds of natural and anthropogenic origin in northeast Atlantic Greenland shark (Somniosus microcephalus). Environmental Toxicology and Chemistry 2010, 29 (12), 2653-2659.

27. Fisk, A. T.; Tittlemier, S. A.; Pranschke, J. L.; Norstrom, R. J., Using anthropogenic contaminants and stable isotopes to assess the feeding ecology of greenland sharks. Ecology 2002, 83 (8), 2162-2172.

28. Cotronei, S.; Pozo, K.; Audy, O.; Přibylová, P.; Corsolini, S., Contamination Profile of DDTs in the Shark Somniosus microcephalus from Greenland Seawaters. Bulletin of Environmental Contamination and Toxicology 2018, 101 (1), 7-13. 